\title{
Influence of contact angle boundary condition on CFD simulation of $\mathrm{T}$-junction
}

\author{
S. Arias - A. Montlaur
}

\begin{abstract}
In this work, we study the influence of the contact angle boundary condition on 3D CFD simulations of the bubble generation process occurring in a capillary T-junction. Numerical simulations have been performed with the commercial Computational Fluid Dynamics solver ANSYS Fluent v15.0.7. Experimental results serve as a reference to validate numerical results for four independent parameters: the bubble generation frequency, volume, velocity and length. CFD simulations accurately reproduce experimental results both from qualitative and quantitative points of view. Numerical results are very sensitive to the gas-liquidwall contact angle boundary conditions, confirming that this is a fundamental parameter to obtain accurate CFD results for simulations of this kind of problems.
\end{abstract}

Keywords Microgravity - Two-phase flows · Bubble generation · T-junction . Computational Fluid Dynamics (CFD) · Contact angle

\section{Introduction}

Gas-liquid two-phase flows dominated by capillary forces occur in countless modern industrial applications of interest to the designers of space-based as well as terrestrial equipment. Examples can be found in normal gravity examples such as mixing process, chemical reactions, emulsion technology, materials synthesis, pipeline systems, medical science, personal care products, etc. These operations are critical to a wide variety of industries such as petroleum, pharmaceutical,

\section{F. S. Arias}

Escola d'Enginyeria de Telecomunicació i Aeroespacial de Castelldefels, Universitat Politècnica de Catalunya, c/ Esteve Terradas 5, 08860 Castelldefels, Barcelona (Spain)

Tel.: +34 934134129

E-mail: santiago.arias@upc.edu

ORCID: 0000-0001-9233-0178

\section{S. A. Montlaur}

Escola d'Enginyeria de Telecomunicació i Aeroespacial de Castelldefels, Laboratori de Càlcul Numèric (www-lacan.upc.edu), Universitat Politècnica de Catalunya, c/ Esteve Terradas 5, 08860 Castelldefels, Barcelona (Spain)

ORCID: 0000-0002-0243-668X 
mining, biological, and chemical. Similar operations will need to be performed in space and on planetary bodies, if we are to achieve our goals of human space exploration. Indeed, gas-liquid two-phase flows intervene as well in space-based systems such as life-support systems for human exploration, thermal management systems, propulsion systems, chemical contactors, space bioreactors, etc (Ostrach, 1988; McQuillen et al., 1998; Baroud \& Willaime, 2004; Guo et al., 2017). A better understanding and an improvement of two-phase flows generation techniques are thus mandatory for the optimal development of these technologies, both in normal and reduced-gravity environments. Regarding microgravity conditions, several methods have been proposed in the past to fulfill this goal, aiming to generate and control two-phase flows in a more accurate way (Pampering \& Rath, 1995; Bhunia et al., 1998; Forrester et al., 1998; Di Marco et al., 2003; Iacona et al., 2006). T-junction bubble generators have arisen as an efficient method providing trains of bubbles, immersed into a continuous liquid, with small dispersion in bubble size (Carrera et al., 2008; Arias et al., 2009, 2010).

Very recently, the authors of the present work introduced a 3D numerical study of the bubble generation process into a T-junction, obtained with the commercial Computational Fluid Dynamics (CFD) solver ANSYS Fluent v15.0.7 (Arias \& Montlaur, 2017). In this study, numerical simulations were compared with experimental data, reproducing the same conditions. Numerical data agreed with experimental results from a qualitative point of view, but showed quantitative discrepancies. Obtained numerical results depended on the gas-liquid-wall contact angle boundary conditions. Consequently, CFD and experimental results never agreed simultaneously for time scale results (bubble generation frequency) and properties of bubbles detachment (bubble volume and velocity), and the real impact of the value of the gas-liquid-wall contact angle remained an open question. This is indeed a complex paradoxical problem, since the interface is a contact line moving along a no-slip solid surface, which has led to several recent studies (Malekzadeh \& Roohi , 2015; Yong-Qiang et al., 2017). As mentioned by Hirt \& Brethour (2001), in the Volume of Fluid (VOF) algorithm approach, which will be used here, dynamic contact angles are not specified but computed as part of the solution via the finite volume method. They arise automatically from the basic balance of forces on which the numerical method is built. This is one of the several practical advantages of this modeling approach. Nevertheless, the numerical description of contact angles suffers from artificial diffusion (Schonfeld \& Hardt, 2009). Huh \& Scriven (1971) suggested that the viscous dissipation becomes very strong near a moving contact line, being dominant with respect to inertial or capillary effects in that region. Afkhami et al. (2009) found that this intense dissipation can curve the interface dramatically near the contact line, which can cause the contact angle to differ significantly from the measured interface angle. In any case, the study and selection of the adequate value of the contact angle for 3D-CFD simulations of multiphase flows in a channel remains a challenging problem, motivating and demanding further work such as the one presented here. For example, Rosengarten et al. (2006) implemented the contact angle within the surface tension model in the solver. The fluid contact angle at the wall was used to rotate the surface normal in the cells adjacent to the wall and thus to adjust the curvature and interface shape near the wall, following a method first described by Brackbill et al. (1992). Shi et al. (2014) used the free energy model of the Lattice Boltzmann method and showed that the droplet size and shape in T-junction 
microchannel depend on the wetting property of channel walls. Here, and given the previously commented difficulty to account for the physics of moving contact lines, the objective is to set the contact angle as a boundary condition.

This paper is a continuation of the study by Arias \& Montlaur (2017), aiming to tackle this issue, by explicitly discussing and quantifying the importance of the value of the gas-liquid-wall contact angle. Section 2 briefly recalls the T-junction problem statement, identifying the relevant dimensionless numbers characterizing the bubble generation process. Section 3 summarizes the experimental and numerical metrics and methodology. Section 4 analyzes the influence of the contact angle boundary conditions, whereas new numerical data are provided and compared with experimental results in section 5 . The main conclusions are outlined in section 6 .

\section{Problem statement}

The experiment considered here consists in generating trains of bubbles in a capillary T-junction. Air and water are injected in perpendicular directions into $1 \mathrm{~mm}$ internal diameter capillaries, see figures 7 and 8 . The liquid capillary is the main channel, with a total length of $10 \mathrm{~mm}$. The gas capillary intersects perpendicularly to the liquid one. Air and water are considered incompressible and under isothermal conditions. Standard values at $25^{\circ} \mathrm{C}$ are used for density $\left(\rho_{G}=1.225 \mathrm{~kg} / \mathrm{m}^{3}\right.$ and $\rho_{L}=10^{3} \mathrm{~kg} / \mathrm{m}^{3}$, for gas and liquid respectively), viscosity $\left(\mu_{G}=10^{-5}\right.$ Pa.s and $\mu_{L}=10^{-3}$ Pa.s $)$ and surface tension $(\sigma=0.072 \mathrm{~N} / \mathrm{m})$.

A detailed description of the experimental setup can be found in (Arias \& González-Cinca, 2016). Gas and liquid volumetric flow rates $\left(Q_{G}\right.$ and $Q_{L}$, respectively) can be accurately controlled, producing bubbles with high regularity. Results will be presented in section 5 as a function of the superficial velocities (instead of the volumetric flow rates). The gas and liquid superficial velocities are defined as $U_{S G}=Q_{G} / A$ and $U_{S L}=Q_{L} / A$, where $A$ is the capillary cross-section area. Moreover, the actual gas and liquid velocities are defined as $U_{G}=Q_{G} /(\alpha A)$ and $U_{L}=Q_{L} /((1-\alpha) A)$, where $\alpha$ is the volume average void fraction (Arias \& Montlaur, 2017). Therefore, the relationships between the superficial and the actual velocities are $U_{G}=U_{S G} / \alpha$ and $U_{L}=U_{S L} /(1-\alpha)$.

Although new experimental results are presented in this work, the range of $U_{S G}$ and $U_{S L}$ analyzed here remains the same as in (Arias \& Montlaur, 2017), as well as the dimensionless numbers that characterize the problem. Table 1 summarizes these values. $B o$ is the Bond number, defined as $\rho_{L} g \phi_{c}^{2} / \sigma$, with the gravitational acceleration $g$ and the capillary internal diameter $\phi_{c}$. We is the Weber number, defined as $W e=\rho_{G} \phi_{c} U_{G}^{2} / \sigma$, for a gas velocity $U_{G}$. Re is the Reynolds number, defined as $R e=\rho_{L} \phi_{c} U_{M} / \mu_{L}$, where $U_{M}$ is the mixture superficial velocity, sum of the gas and liquid superficial velocities. Finally, $C a$ is the capillary number, defined as $C a=\mu_{L} U_{S L} / \sigma$. As shown by the values in table 1 , gravity plays a negligible role in the bubble generation process with respect to capillary effects $(B o<0.29$, (Suo \& Griffith, 1964)), and capillary forces overcome inertial forces $(W e<2$, (Rezkallah, 1996)). Values of Re correspond to flows under laminar conditions, and surface tension effects are greater than the liquid viscous effects $\left(C a<10^{-2}\right)$. Consequently, the present analysis can be assumed as gravity independent $(g=$ 
Table 1 Characteristic experimental values

\begin{tabular}{lllllll}
\hline & $\begin{array}{l}U_{S G} \\
{[\mathrm{~m} / \mathrm{s}]}\end{array}$ & $\begin{array}{l}U_{S L} \\
{[\mathrm{~m} / \mathrm{s}]}\end{array}$ & Bo & $W e$ & $R e$ & $C a$ \\
\hline $\begin{array}{l}\text { Minimum } \\
\text { value }\end{array}$ & 0.068 & 0.106 & 0.139 & $4.3 \times 10^{-4}$ & 167 & $1.5 \times 10^{-3}$ \\
$\begin{array}{l}\text { Maximum } \\
\text { value }\end{array}$ & 0.473 & 0.531 & 0.139 & $2.2 \times 10^{-2}$ & 1000 & $7.4 \times 10^{-3}$ \\
\hline
\end{tabular}

$0 \mathrm{~m} / \mathrm{s}^{2}$ ), and the bubble generation phenomena can be considered laminar and mainly controlled by the surface tension.

\section{Methodology}

\subsection{Experimental procedure and metrics}

Results of a total of 15 experiments are compared with numerical simulations, with same gas and liquid superficial velocities and fluid physical properties used in both cases. Experiments were performed at 3 different liquid superficial velocities $(0.106$, 0.318 and $0.531 \mathrm{~m} / \mathrm{s}$ ) while increasing the gas superficial velocity (within the range $0.068-0.473 \mathrm{~m} / \mathrm{s}$ ). For each pair of velocities $U_{S L}-U_{S G}$, images were taken with a high-speed camera at 4000 frames per second (f.p.s), once the flow had stabilized. Only two types of flow regimes were obtained, bubble and slug flow regimes. Bubble flow regime occurs when bubble volume $V_{B}$ is smaller than the volume of a sphere $V_{S}$ with a diameter equal to the capillary internal diameter $\phi_{c}$, and slug flow regime in the other case $\left(V_{B}>V_{S}\right)$. Both types of flow patterns are intermittent flows in which the gas phase is dispersed into the liquid phase forming independent bubbles. The bubble generation frequency for a single bubble is defined as $f_{i}=1 / T_{f i}, T_{f i}$ being the total time required to form and detach a single bubble, denoted with the subscript $i$. $T_{f i}$ was computed by counting the number of frames required to form the bubble $i$, that is, $T_{f i}=$ \#frames $/ 4000$ f.p.s. The bubble volume of each single bubble $V_{B i}$ was estimated taking into account that $Q_{G}=f_{i} \cdot V_{B i}$, and then that $V_{B i}=Q_{G} \cdot T_{f i}$. The bubble (or gas) velocity $U_{G i}$ was measured directly over the calibrated images, by considering the displacement of the foremost part of a single bubble. Finally the bubble length $L_{B i}$ was also measured over calibrated images. For each pair $U_{S L}-U_{S G}$, the average values of these four parameters were estimated over a large enough sample of $n$ bubbles. Standard deviations calculated for each experimental value accounted for uncertainty. They have been plotted as error bars in figures 9-12, representing an improvement of the experimental data with respect to (Arias \& Montlaur, 2017). For the sake of clarity, error bars smaller than the corresponding symbols in the graphs are not represented.

The same four parameters were obtained in the numerical simulations. Figure 1 shows an example of data obtained as a post-process of the CFD simulations, that is, the fraction of air as a function of time at two cross-sections of the domain (at 7 and $8 \mathrm{~mm}$ from the beginning of the tube). The frequency is computed as the inverse of the time between two consecutive bubbles when crossing the same cross-section, that is, as $1 / T_{f}$. The bubble velocity is calculated as the distance between cross-sections at 7 and $8 \mathrm{~mm}$ divided by the time $T_{S}$, needed for the 


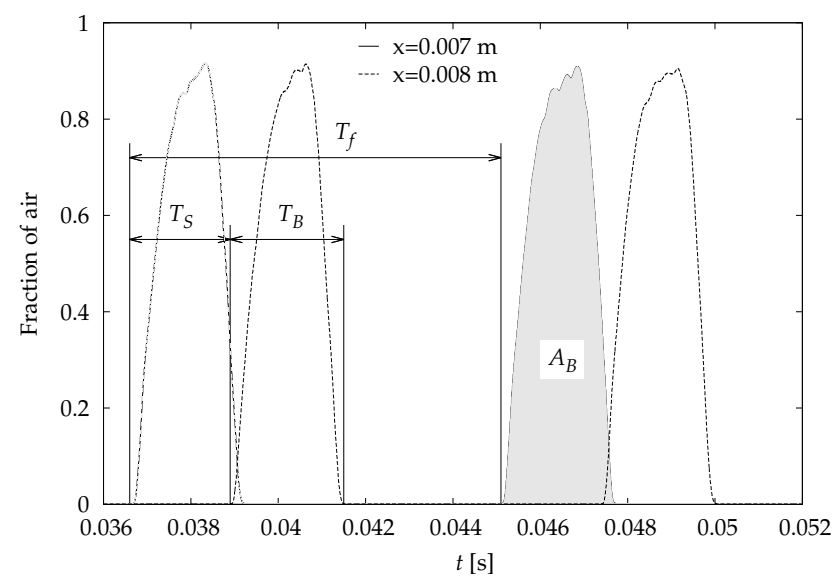

Fig. 1 Example of CFD post-processed results obtained from the graph of fraction of air as a function of time, for $U_{S L}=0.318 \mathrm{~m} / \mathrm{s}$ and $U_{S G}=0.081 \mathrm{~m} / \mathrm{s}$

bubble to travel this distance. The bubble volume is computed by integrating the total fraction of air of a bubble over time $\left(A_{B}\right)$ and multiplying it by its velocity times the capillary cross-section area. The bubble length is calculated as the time between the foremost and rearmost times of a bubble $\left(T_{B}\right)$ multiplied by the bubble velocity.

\subsection{Numerical simulations}

All numerical simulations use the commercial CFD solver ANSYS Fluent v15.0.7. Both fluids are modeled as immiscible ones by using a three-dimensional Volume of Fluid (VOF) method and tracking the volume fraction of each fluid, as well as the interface between the phases, throughout the domain. An explicit formulation is used for the VOF model, the PRESTO! (PREssure STaggering Option) scheme for pressure interpolation (Ansys, 2014), a second-order upwind scheme for spatial discretization and a first-order implicit scheme for the transient formulation. Regarding the boundary conditions, air and water inlets are considered as velocity inlets, with the corresponding values of $U_{S G}$ and $U_{S L}$ in each case of study. The outlet of the T-junction is set up as a pressure outlet. All walls are treated as no-slip smooth walls with identical constant contact angle, which is the key point of the present study, defining it as the angle between the wall and the tangent to the interface at the wall (measured from the liquid toward the gaseous phase). All gas and liquid physical properties are set to the same values as in the experiments. The gravity value is set to 0 , accordingly to the experimental small value of the Bond number. All details concerning this CFD solver and the settings used for these numerical simulations can be found in (Arias \& Montlaur, 2017).

The computational mesh is composed of 413000 elements. This mesh is a hybrid of tetrahedral (in the core of the domain) and hexahedral (close to the walls) elements. The value $\Delta t=5 \times 10^{-6} \mathrm{~s}$ is selected as a time step for all the simulations. Again, more details about the chosen mesh and time step and their validation can be found in (Arias \& Montlaur, 2017). 


\section{Contact angle study}

We next denote $\theta_{V}$ the value of the gas-liquid-wall contact angle boundary condition on the vertical capillary, $\theta_{H}$ on the horizontal one, and simplify the notation to $\theta$ when $\theta_{V}=\theta_{H}$. In a previous work, Arias \& Montlaur (2017) considered a constant $\theta_{V}$. The horizontal channel was maintained perfectly hydrophilic, that is, $\theta_{H}=0^{\circ}$, following previous set-up from Arias et al. (2012), and given that experimental results showed no attachment of the gas at this channel. Additionally, two-dimensional numerical simulations performed in (Arias et al., 2012) found that the condition $\theta_{H}=0^{\circ}$ was essential in order to generate bubbles with a realistic shape (non-attached to this channel). In (Arias \& Montlaur, 2017), numerical results were found to be very sensitive to the value of $\theta_{V}$. Furthermore, no single value of $\theta_{V}$ could provide simultaneously close-to-experimental results for the bubble frequency, velocity and volume, not even for a single pair $U_{S L}-U_{S G}$. In fact, it was shown that when the value of $\theta_{V}$ increased, the pinch-off region moved downstream, producing a detachment mechanism that differed from the one observed in the experiments (see figure 3 in (Arias \& Montlaur, 2017)). Eventually, values of $\theta_{V}=25^{\circ}$ and $\theta_{H}=0^{\circ}$ were fixed in all numerical simulations, given that this configuration provided the best resemblance with experimental pinch-off, despite leading to some numerical error.

In the current study, we cancel the perfect hydrophilic boundary condition by imposing the same (non-zero) value of the contact angle in the horizontal channel as in the vertical one. This change of boundary condition has been motivated by the previously commented impossibility of reaching good agreement by only tuning $\theta_{V}$. Furthermore, it is also found more logical from a physical point of view, since the same contact angle $\theta$ is now imposed on every wall, meaning that the wall boundary condition is now continuous in the critical region of the $\mathrm{T}$ junction where pinch-off occurs. Different values of contact angles (within the range $0-90^{\circ}$ ) were tested for each pair $U_{S L}-U_{S G}$, until finding a value that simultaneously provided close-to-experimental results (for the bubble frequency, volume, velocity and length) and a realistic pinch-off process. Figure 2 presents the values $\theta$ that best fulfill these requirements for each pair $U_{S L}-U_{S G}$ studied here. The contact angle is plotted as a function of the actual liquid velocity $U_{L}$, which is computed by canceling $\alpha$ in the definitions of $U_{L}$ and $U_{G}$ (see section 2):

$$
U_{L}=\frac{U_{S L}}{1-\frac{U_{S G}}{U_{G}}},
$$

where values of $U_{G}$ come from experiments (see section 5.3). Figure 2 shows that values in the range of $\theta=25-30^{\circ}$ are optimal for $U_{L}<0.415 \mathrm{~m} / \mathrm{s}$ and $U_{L}>0.756 \mathrm{~m} / \mathrm{s}$. This confirms that the $25^{\circ}$-value imposed in (Arias \& Montlaur, 2017) in the vertical channel was correct, but, and as it will be further commented in the following sections, it had to be also imposed in the horizontal channel. No suitable constant value of $\theta$ was found in the range $0.415<U_{L}<0.756 \mathrm{~m} / \mathrm{s}$. Within this range, fine-tuning of $\theta$ should be made for every particular case; nevertheless, further tests have proved that imposing a constant value of $\theta=40^{\circ}$ (calculated as the average within this range), would not generate any substantial variation in the solution. The observed discontinuity of the optimal value of $\theta$ has no trivial explanation, and no correlation with any physical behavior or experi- 


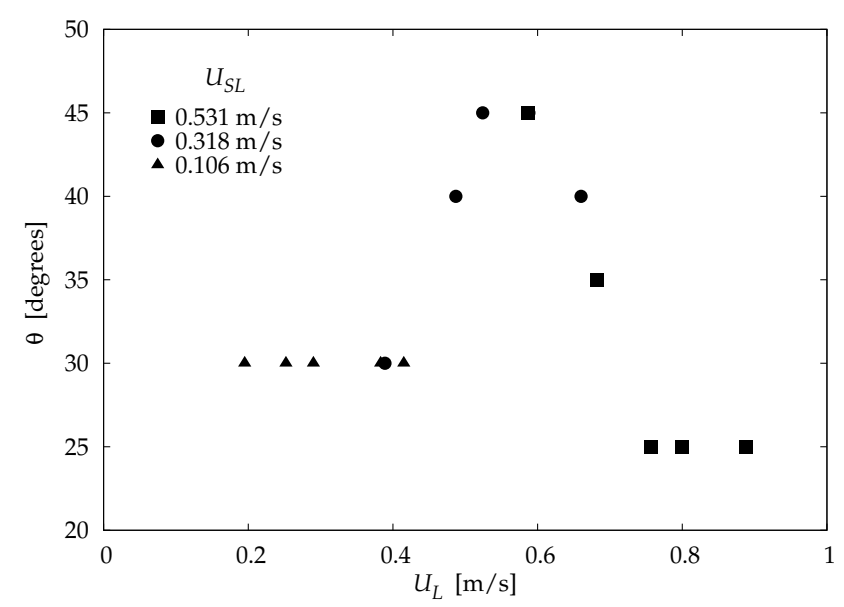

Fig. 2 Contact angle as a function of the actual liquid velocity.

mental tendency has been found. Further studies could try to provide a suitable answer to this particular subject.

Figure 3 shows bubbles just after detachment for contact angles of $25^{\circ}, 35^{\circ}$, $45^{\circ}, 90^{\circ}$ (for $U_{S L}=0.531 \mathrm{~m} / \mathrm{s}$ and $U_{S G}=0.178 \mathrm{~m} / \mathrm{s}$ ). The contact angle is indicated in the upper right corner. The corresponding experimental picture is also included (between the two most similar numerical simulations). The main effect appearing when disabling the perfectly hydrophilic condition, that is, now $\theta_{H}=\theta_{V}$, is that the gas can now be attached to the horizontal channel. Gas attachment only occurs in the pinch-off zone for small contact angles (such as $25^{\circ}$ and $35^{\circ}$ ), but also appears further away from this zone for larger values (with $45^{\circ}$, and especially in the extreme case of $90^{\circ}$ ). It plays a fundamental role during the bubble generation process: as the gas thread connecting the bubble to the single-phase gas adheres to the horizontal channel in the pinch-off zone, the bubble formation process slows down. This effect decreases the value of the bubble generation frequency, while increasing the bubble volume and length. Note that, when there is no attachment at all, bubbles have been found to be generated faster than in experiments, resulting in larger frequencies, as found in (Arias \& Montlaur, 2017). However, if the value of the contact angle is too high, bubbles become partially or totally attached to the horizontal channel outside this pinch-off zone, which results in a non-realistic physical behavior as clearly shown in figure 3 for the extreme value of $\theta=90^{\circ}$. Therefore, the selection of $\theta$ relies on a trade-off between the necessity of getting slight adherence in the pinch-off region, and the requirement of having no attachment further away from this zone.

Figure 4 shows the bubble generation frequency as a function of $\theta_{V}$ (for $U_{S L}=$ $0.531 \mathrm{~m} / \mathrm{s}$ and $\left.U_{S G}=0.178 \mathrm{~m} / \mathrm{s}\right)$, for numerical simulations with either $\theta_{H}=\theta_{V}$ or $\theta_{H}=0^{\circ}$. Note that the plain lines in figures 4 and 5 aim to help comparing these two cases, but do not correspond to any interpolation. Numerical simulations with $\theta_{H}=\theta_{V}$ generate bubbles at a smaller rate (larger times are required to form a single bubble), producing smaller frequencies, as previously discussed. In addition, figure 4 clearly shows how increasing $\theta_{V}$ reduces the frequency. Figure 5 shows bubble volume (normalized with the capillary diameter times the cross- 


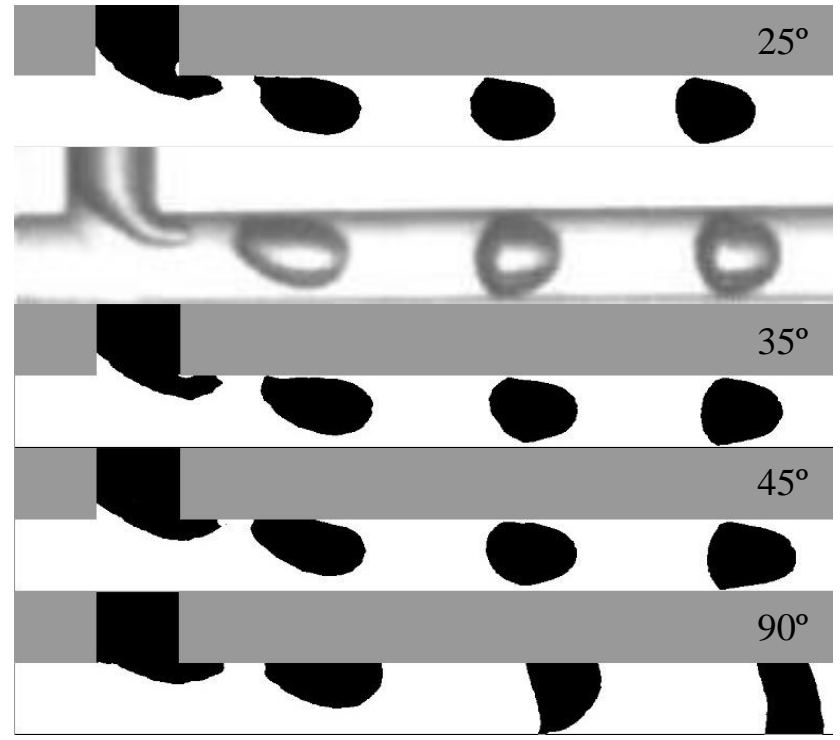

Fig. 3 Bubble generation at the moment just after bubble detachment, for contact angles of $25^{\circ}, 35^{\circ}, 45^{\circ}, 90^{\circ}$ and for the experimental one, for $U_{S L}=0.531 \mathrm{~m} / \mathrm{s}$ and $U_{S G}=0.178 \mathrm{~m} / \mathrm{s}$.

section area) as a function of the contact angle, for the same values of $U_{S L}$ and $U_{S G}$. Bubble volume increases with $\theta_{V}$, since it also increases the time required to form a single bubble. Note that in figures 4 and 5 , the experimental values of frequency $\left(331.2 \mathrm{~s}^{-1}\right)$ and normalized bubble volume $(0.538)$ have been added for reference as horizontal dash lines. It can be seen that when $\theta_{H}=\theta_{V}$, a value of $\theta=35^{\circ}$ is needed to get close to the experimental values. Whereas extremely high and unrealistic values of $\theta_{V}$ would be needed in the perfectly hydrophilic case, $\theta_{V}=90^{\circ}$ for the frequency, and $\theta_{V}=60^{\circ}$ for the normalized bubble volume, confirming the relevancy of the choice of $\theta_{H}=\theta_{V}$.

Finally, the contact angle does not have any major impact on the bubble velocity. Figure 6 shows how several numerical simulations, with different contact angles, provide values of the velocities close to the experimental one $(0.802 \mathrm{~m} / \mathrm{s})$, independently of the value of the contact angle (and whether the horizontal channel is perfectly hydrophilic or not). This is a logical result since the velocity is measured in the region of the T-junction where the gas is fully detached. The only point that is remarkably far away from the experimental result corresponds to the case with $\theta=90^{\circ}$, in which the bubble is completely attached to the horizontal channel (non-physically realistic case), see figure 3 . This artificially imposed gas attachment slows down the bubble displacement along the channel, reducing the bubble velocity.

\section{Results and discussion}

Numerical simulations satisfactorily reproduce the trains of bubbles obtained in experiments. Figures 7 and 8 show examples of bubble and slug flow regimes, respectively. These figures show the formation and detachment of one single bubble, 


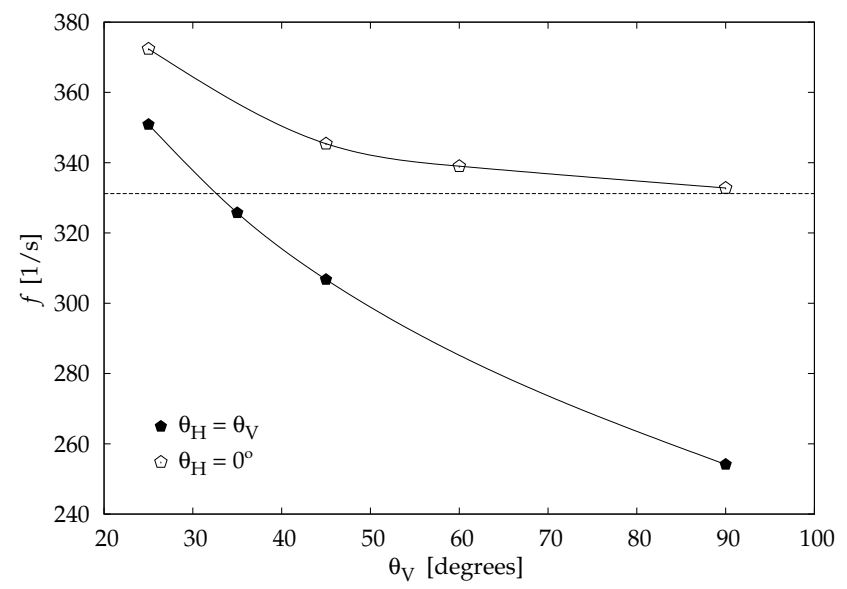

Fig. 4 Bubble generation frequency as a function of the contact angle, for $U_{S L}=0.531 \mathrm{~m} / \mathrm{s}$ and $U_{S G}=0.178 \mathrm{~m} / \mathrm{s}$.

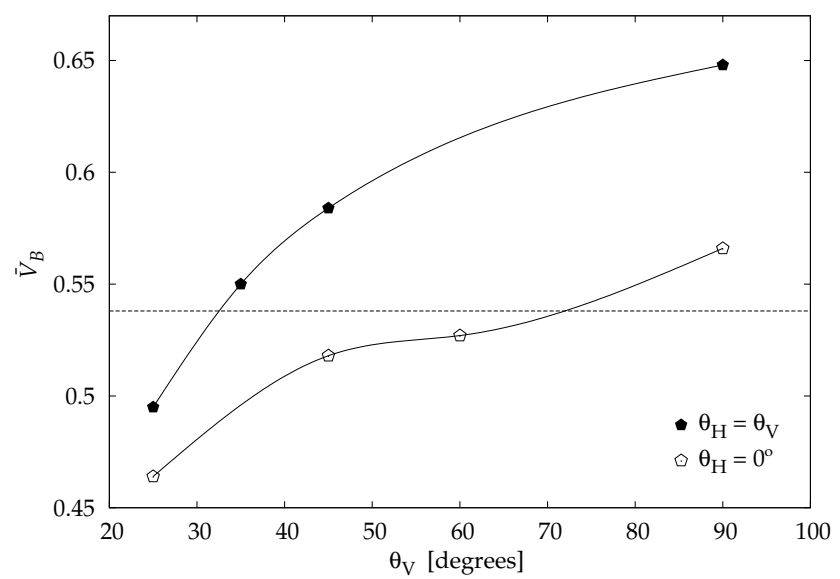

Fig. 5 Normalized bubble volume as a function of the contact angle, for $U_{S L}=0.531 \mathrm{~m} / \mathrm{s}$ and $U_{S G}=0.178 \mathrm{~m} / \mathrm{s}$.

showing several stages during the bubble generation. Contrary to the numerical results presented in (Arias \& Montlaur, 2017) (see figures 5-7 of this reference), the time $T_{f}$ required to form and detach a bubble is now the same in experiments and numerical simulations: 3.0 milliseconds in figure 7 (0.6 millisecond for each step), and 2.75 milliseconds (0.55 millisecond for each step) in figure 8 .

Bubble shapes are also adequately reproduced. Bubbles smaller than the capillary diameter are slightly deviated away from the spherical shape due to the shear stresses exerted by the liquid, see figure 7 . Bubbles bigger than the capillary diameter tend to have bullet-shape bodies, rounded at the front part with a mostly flatted rear part, see figure 8. Independently of the shape, the detachment process is simulated in a satisfactory manner in all cases, correctly reproducing the gas thread connecting the bubble to the single-phase gas. After the detachment, the elongated rear part of the bubble (a remnant of the squeezed gas thread) readjusts 


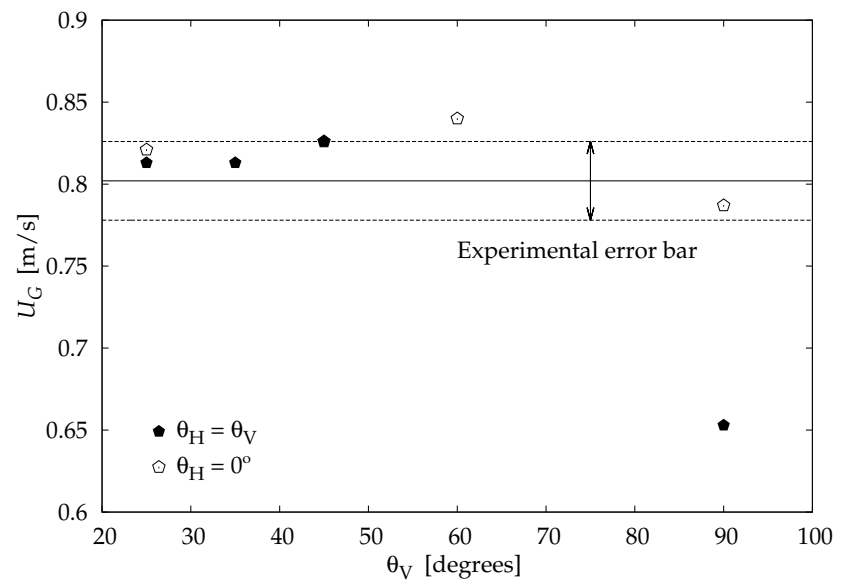

Fig. 6 Bubble velocity as a function of the contact angle, for $U_{S L}=0.531 \mathrm{~m} / \mathrm{s}$ and $U_{S G}=$ $0.178 \mathrm{~m} / \mathrm{s}$.
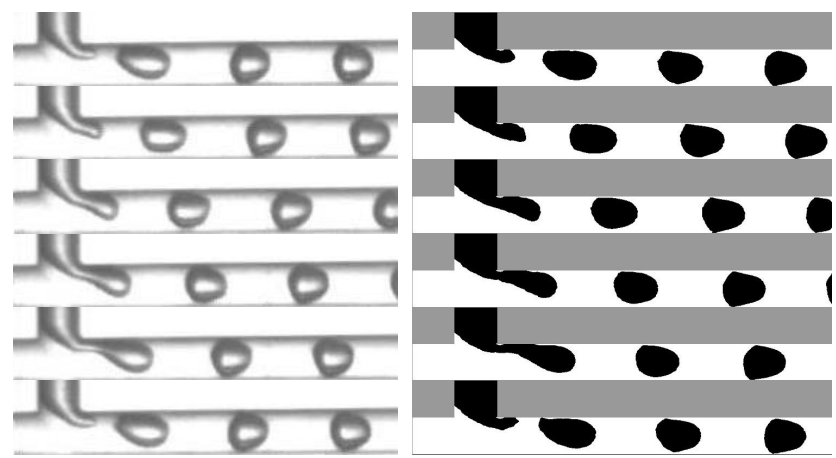

Fig. 7 Bubble flow in (left) experiments and (right) numerical simulations, for $U_{S L}=$ $0.531 \mathrm{~m} / \mathrm{s}$ and $U_{S G}=0.178 \mathrm{~m} / \mathrm{s}$.

elastically, because of the effect of the surface tension, in order to reduce the total bubble surface.

Next four sections validate the numerical simulations in a quantitative way by comparing their results regarding bubble frequency, volume, velocity and length with experimental data. In figures 9-12, numerical simulations have been plotted with solid symbols (and noted as Sim.), and experiments with empty symbols (and noted as Exp.). As commented in (Arias \& Montlaur, 2017), errors due to the selected mesh size and time step are within a relatively small margin $(\leq 3.1 \%$ for all selected parameters). From a quantitative point of view, these errors are similar to the corresponding standard deviations found in experiments. Therefore, in the following sections, numerical errors obtained within this margin can be considered as negligible. 


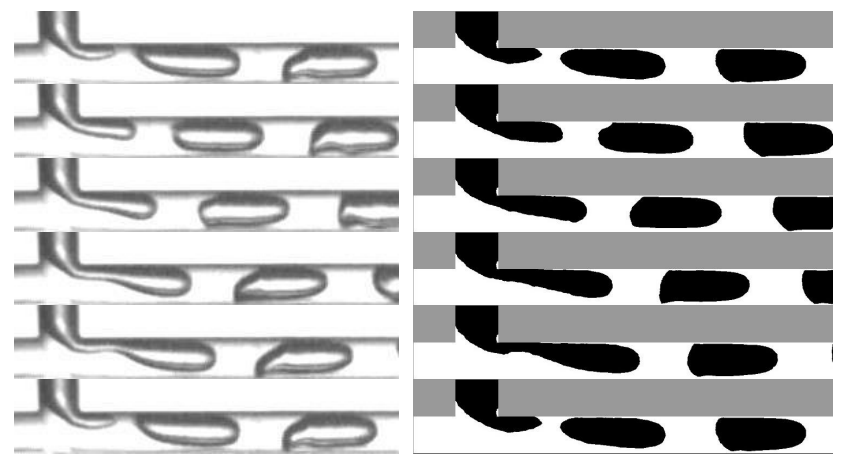

Fig. 8 Slug flow in (left) experiments and (right) numerical simulations, for $U_{S L}=0.531 \mathrm{~m} / \mathrm{s}$ and $U_{S G}=0.469 \mathrm{~m} / \mathrm{s}$.

\subsection{Bubble generation frequency}

Figure 9 shows the bubble generation frequency as a function of the superficial gas velocity, with data from experiments and numerical simulations. The frequency first follows a close-to-linear tendency (for very low gas flow rate), that progressively curves until reaching a saturation value (for larger gas flow rates). A simple exponential fit for this behavior is plotted in figure 9 (for each liquid superficial velocity), whose details can be found in (Arias \& Montlaur, 2017). Numerical simulations satisfactorily describe this behavior and agree qualitatively and quantitatively with experimental results. In some cases, experimental and numerical points even overlap. Errors in frequency for numerical results with respect to experiments have an average value of $3.2 \%$, with a standard deviation of $2.6 \%$, which are values close to the precision margin of the chosen mesh and time step. Note that, the errors obtained in (Arias \& Montlaur, 2017) (considering only the same numerical simulations) were up to $17.6 \%$ for the average frequency value and $8.7 \%$ for their standard deviation, which clearly shows the improvement in the numerical results obtained when imposing the $\theta_{H}=\theta_{V}$ boundary condition.

\subsection{Bubble volume}

Figure 10 shows results from experiments and numerical simulations of the bubble volume, which has been normalized with the capillary diameter times the crosssection area, that is, $\bar{V}_{B}=U_{S G} / f \phi_{c}$. In the experiments, the average value of the bubble volume standard deviations was found to be 0.055 , with a maximum value of 0.156 (corresponding to the case with the largest value of the bubble volume, $U_{S L}=0.106 \mathrm{~m} / \mathrm{s}$ and $\left.U_{S G}=0.471 \mathrm{~m} / \mathrm{s}\right)$. These small values of experimental dispersion in bubble volume show that the bubble formation process was conducted with high regularity and periodicity in all experiments. Numerical simulations agree qualitatively with experimental results, bubble volume increasing with the gas superficial velocity. Numerical errors for bubble volumes are of $2.8 \%$ in average, which is again within the mesh and time step precision margin, with a standard deviation of $1.9 \%$, resulting in numerous overlapping points for experiments and numerical simulations in figure 10. Once again, these values of error 


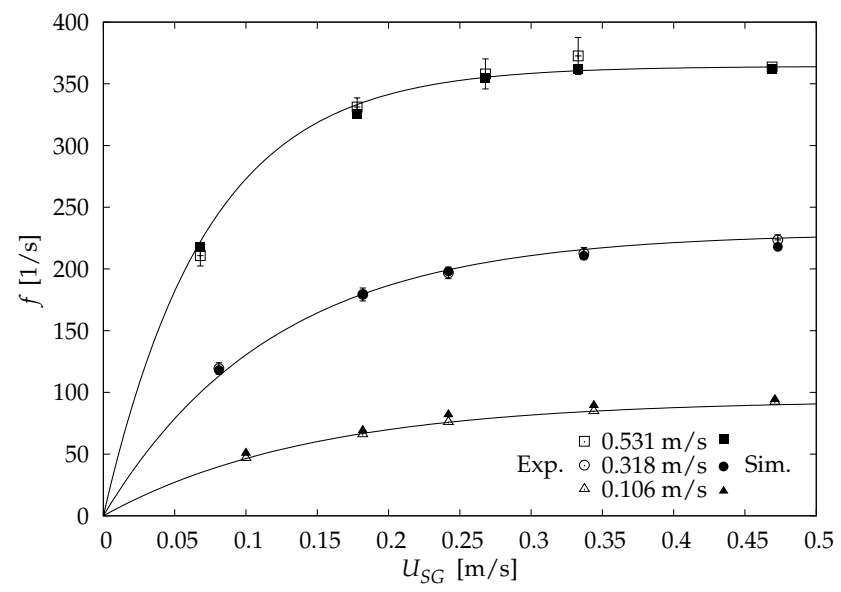

Fig. 9 Bubble frequency as a function of the superficial gas velocity for three different superficial liquid velocities.

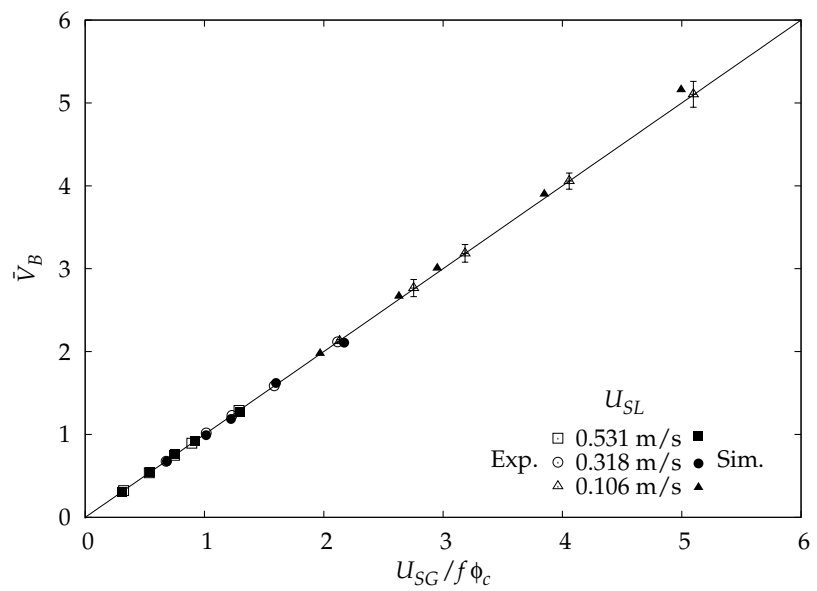

Fig. 10 Normalized bubble volume as a function of $U_{S G} /\left(f \phi_{c}\right)$.

show great improvement with respect to the numerical results presented in (Arias \& Montlaur, 2017), where an average error of $15.0 \%$ for bubble volume and a standard deviation of $6.9 \%$ were obtained.

\subsection{Bubble velocity}

The drift-flux model predicts that under the hypothesis of non-dominant gravitational forces, the bubble velocity must be proportional to the mixture velocity (Zuber \& Findlay, 1969), that is $U_{G}=C_{0} U_{M}$, where $C_{0}$ is called the void fraction distribution coefficient. Figure 11 illustrates this linear tendency. The value of $C_{0}$ has been calculated using a linear fitting for the experimental data, resulting in a value of 1.13 . This value is coherent with the values reported in the related literature for similar two-phase flows (Arias \& Montlaur, 2017). Numerical errors for 


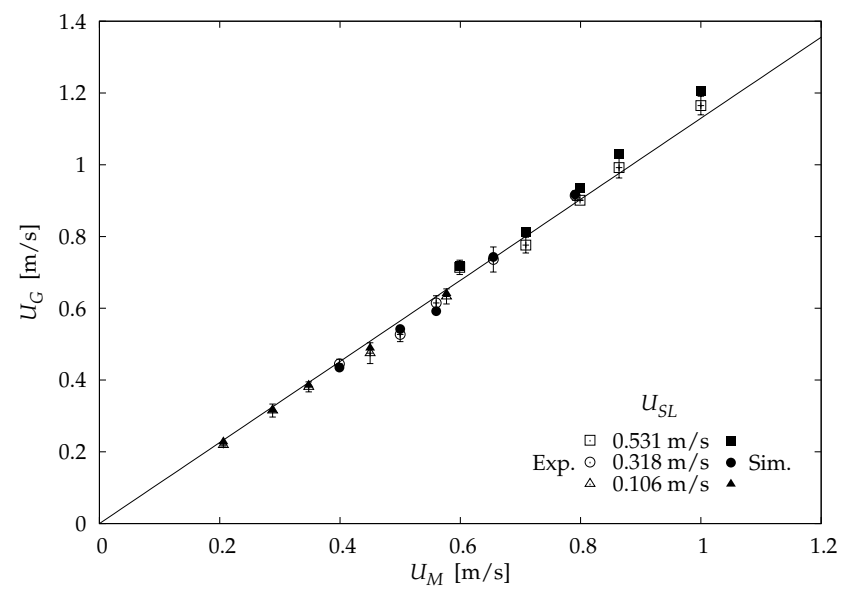

Fig. 11 Bubble velocity as a function of the mixture superficial velocity.

bubble velocity have an average value of $2.2 \%$, with a standard deviation of $1.3 \%$, and again the experimental and corresponding numerical points often overlap in figure 11, improving the errors obtained in (Arias \& Montlaur, 2017) of $5.2 \%$ for the average velocity value and $2.9 \%$ for the standard deviation.

\subsection{Bubble length}

The bubble length, normalized with the capillary diameter, is plotted as a function of $U_{S G} / f \phi_{c}$ in figure 12. Experimental data fit to the linear expression:

$$
\bar{L}_{B}=C_{1}+C_{2} \frac{U_{S G}}{f \phi_{c}}
$$

where $C_{1}=0.62$ and $C_{2}=0.90$ are fitting constants. Equation 2 is similar to the expressions used by Arias \& González-Cinca (2016) for comparable experimental data. As shown in figure 12, the bubble length increases with the gas superficial velocity, following a linear tendency. Equation 2 has also been plotted in figure 12. An average difference of $3.4 \%$ is obtained between experimental and numerical results, with a standard deviation of $1.8 \%$. Though Arias \& Montlaur (2017) had not presented any results concerning the bubble length, it has now been computed in order to quantitatively compare the perfectly hydrophilic and the non-zero boundary conditions. An average error of $9.9 \%$ for the bubble length and a standard deviation of $6.2 \%$ have been found for the perfectly hydrophilic case, showing again the necessity of the $\theta_{H}=\theta_{V}$ boundary condition.

\section{Conclusions}

This paper studies the influence of the gas-liquid-wall contact angle boundary condition on 3D-CFD simulations of the bubble generation process occurring in a capillary T-junction. This paper is a continuation of the previous work of Arias \& 


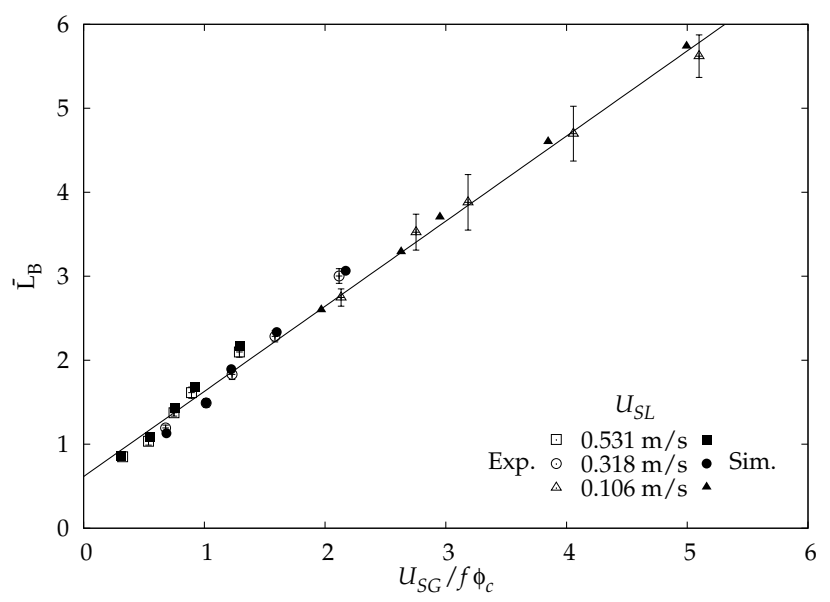

Fig. 12 Normalized bubble length as a function of $U_{S G} / f \phi_{c}$.

Montlaur (2017), which showed the influence of the contact angle but left it as an open question.

The commercial CFD solver ANSYS Fluent v15.0.7 provided numerical simulations of an air/water mixture in a T-junction, and obtained data were validated by comparison with experimental data. Small dispersion in size during the bubble formation process was achieved. Numerical simulations reproduced trains of bubbles with similar physical behavior as the one observed in experiments, accurately reproducing the experimental bubble shapes.

Numerical results showed to be very sensitive to the gas-liquid-wall contact angle boundary condition. In particular, fixing a non-zero value of the contact angle boundary condition on the horizontal wall happened to be of critical importance in order to obtain reliable and close-to-experimental numerical results. The major difference, with respect to (Arias \& Montlaur, 2017), is that in this present work, the same value of contact angle has been imposed on both vertical and horizontal channel walls, now providing much more accurate results. Values of $\theta=30^{\circ}$ for $U_{L}<0.415 \mathrm{~m} / \mathrm{s}, \theta=40^{\circ}$ for $0.415<U_{L}<0.756 \mathrm{~m} / \mathrm{s}$ and $\theta=25^{\circ}$ for $U_{L}>0.756 \mathrm{~m} / \mathrm{s}$ were found to be the most adequate ones. The study also showed that increasing the contact angle value decreases the bubble generation frequency (increasing the bubble volume and length). As for the bubble velocity, it does not depend significantly on the value of the contact angle, as long as the bubbles do not totally adhere to the horizontal channel, which would happen in an extreme and non-realistic case of perpendicular contact angle.

Results on bubble generation frequency, volume, velocity and length were compared between 3D-CFD and experimental data. Relative errors and standard deviations of these results prove that the $\theta_{H}=\theta_{V}$ boundary condition adopted in this work is essential, and that it greatly improves the results presented in (Arias \& Montlaur, 2017) (with $\theta_{H}=0^{\circ}$ ). Therefore, the contact angle boundary condition is a key parameter to obtain accurate results for numerical simulations of two-phase flows in a capillary. 
Acknowledgements This work has been financially supported by the Spanish Ministerio de Economía y Competitividad, Secretaría de Estado de Investigación, Desarrollo e Innovación (Project numbers ESP2016-79196-P and MTM2013-46313-R) and the Generalitat de Catalunya (Grant number 2017-SGR-1278).

\section{References}

Afkhami, S., Zaleski, S., Bussmann M.: A mesh-dependent model for applying dynamic contact angles to VOF simulations. Journal of Computational Physics. 228, 5370-5389 (2009)

ANSYS Academic Research, Release 15.0,7, Help System, ANSYS, Inc. (2014)

Arias, S., Ruiz, X., Ramírez-Piscina, L., Casademunt, J., González-Cinca, R.: Experimental study of a microchannel bubble injector for microgravity applications. Microgravity Sci. Technol. 21, 107-118 (2009)

Arias, S., González-Cinca, R., Ruiz, X., Ramírez-Piscina, L., Casademunt, J.: Characterization of the performance of a minibubble generator in conditions relevant to microgravity. Colloids Surfaces A: Physicochem Eng Aspects. 365, 52-57 (2010)

Arias, S., Legendre, D., González-Cinca, R.: Numerical simulation of bubble generation in a T-junction. Computers and Fluids. 56, 49-60 (2012)

Arias, S., González-Cinca, R.: Analysis of the characteristic lengths in the bubble and slug flow regimes generated in a capillary T-junction. International Journal of Multiphase Flow. 87, 167-174 (2016)

Arias, S., and Montlaur, A.: Numerical study and experimental comparison of two-phase flow generation in a T-junction. AIAA Journal, 55, No. 5, 1565-1574 (2017)

Baroud, C.N., Willaime, H.: Multiphase flows in microfluidics. C. R. Phys. 5, 547555 (2004)

Bhunia, A., Pais, S.C., Kamotani, Y., Kim, I.: Bubble formation in a coflow configuration in normal and reduced gravity. AIChE J. 44, 1499-1509 (1998)

Brackbill, J.U., Kothe, D., Zemach, C.: A continuum method for modeling of surface tension. J. Comput. Phys. 100, 335-354 (1992)

Carrera, J., Ruiz, X., Ramírez-Piscina, L., Casademunt, J., Dreyer, M.: Generation of a monodisperse microbubble jet in microgravity. AIAA J. 46(8), 2010-2019 (2008)

Di Marco, P., Grassi, W., Memoli, G., Takamasa, T., Tomiyama, A., Hosokawa, S.: Influence of electric field on single gas-bubble growth and detachment in microgravity. Int. J. Multiph. Flows. 29, 559-578 (2003)

Forrester, S.E., Rielly, C.D.: Bubble formation from cylindrical, flat and concave sections exposed to a strong liquid crossflow. Chem. Eng. Sci. 53, 1517-1527 (1998)

Guo, Q., Ye, F., Guo, H., Ma, C.F.: Gas/Water and Heat Management of PEMBased Fuel Cell and Electrolyzer Systems for Space Applications. Microgravity Sci. Technol. 29, 4963 (2017)

Hirt, C.W., and Brethour, J.M.: Moving contact lines on rough surfaces. 4th European Coating Symposium, FloSci-Bib17-01 (2001)

Huh, C., Scriven, L.E.: Hydrodynamic model of steady movement of a solid/liquid/fluid contact line. Journal of Colloid and Interface Science, 35 (1), 85-101 (1971) 
Iacona, E., Herman, C., Chang, S., Liu, Z.: Electric field effect on bubble detachment in reduced gravity environment. Exp. Therm. Fluid Sci. 31, 121-126 (2006)

McQuillen, J., Colin, C., Fabre, J.: Ground-based gas-liquid flow research in microgravity conditions: state of knowledge. Space Forums. 3, 165-203 (1998)

Malekzadeh, S., Roohi, E.: Investigation of Different Droplet Formation Regimes in a T-junction Microchannel Using the VOF Technique in OpenFOAM. Microgravity Sci. Technol. 27, 231243 (2015)

Ostrach, S.: Industrial processes influenced by gravity. NASA CR-182140, C21066-G (1988)

Pampering, O., Rath, H.J.: Influence of buoyancy on bubble formation at submerged orifices. Chem. Eng. Sci. 50, 3009-3024 (1995)

Rezkallah, K. S.: Weber number based flow-pattern maps for liquid-gas flows at microgravity. International Journal of Multiphase Flow. 22 (6), 1265-1270 (1996)

Rosengarten, G., Harvie, D.J.E., Cooper-White, J.: Contact angle effects on microdroplet deformation using CFD. Applied Mathematical Modelling, 30, 1033-1042 (2006)

Schönfeld, F., and Hardt, S.: Dynamic contact angles in CFD simulations. Computers \& Fluids. 38, 757-764 (2009)

Shi, Y., Tang, G.H., Xia, H.H.: Lattice Boltzmann simulation of droplet formation in T-junction and flow focusing devices. Computers \& Fluids. 90, 155163 (2014)

Suo, M., Griffith, P.: Two-phase flow in capillary tubes. Journal of Basic Engineering. 86, 576-582 (1964)

Yong-Qiang, L., Wen-Hui, C., Ling, L.: Numerical Simulation of Capillary Flow in Fan-Shaped Asymmetric Interior Corner Under Microgravity. Microgravity Sci. Technol. 29, 6579 (2017)

Zuber, N., Findlay, J.: Average Volumetric Concentration in Two-Phase Systems. Journal of Heat Transfer. 87 (4), 453-468 (1969) 